\title{
Analysis on Prediction of Customer Purchasing Decisions using Machine Learning
}

\author{
Nehal Raj, Rajneesh Kumar Singh, Satyajee Srivastava
}

\begin{abstract}
In our day-to-day life, everyone settles on choices on whether to purchase an item or not. In a couple of cases, the choice depends on cost however on numerous occasions the buying choice is more intricate, still, numerous other reasons may be cogitated prior to the last decision is take. Within large-scale industries, understanding existing consumer's purchasing behavior towards the product is more important to drive a business to the next level. In the context to expand the business on a large scale understanding, the customer interest is more important. To understand the behavior of customers and their interest in the products we need some new technologies and a large amount of data. In this paper we present a progression of examinations, investigate and analyze the exhibitions of various ML strategies, and talk about the meaning of the discoveries with regards to public arrangement and purchaser buying choice. Utilizing an enormous certifiable informational collection (which will be unveiled after the distribution of this work), we present a progression of examinations, dissect and look at the exhibitions of various ML procedures, and talk about the meaning of the discoveries with regards to public strategy and consumer buying Decision.
\end{abstract}

Keywords : Purchasing, Clustering, Datasets, Random Forest, Naïve Bayes Classifier.

\section{INTRODUCTION}

Perhaps the most widely recognized monetary choices that every one of us makes on an almost everyday schedule include the buying of different items and merchandise. At times, the choice on whether to make a buy depends to a great extent on cost however in numerous cases, the buying choice is more perplexing, with a lot more contemplations influencing the decision cycle before the last commitment is made. Retailers comprehend this well and endeavor to utilize it with an end goal to acquire an edge in a profoundly serious market. In particular, with an end goal to make buying almost certain, as well as adjusting the saleability and benefit in setting the selling cost of an item, organizations as often as possible acquaint extra components with the offer which are

Manuscript received on March 10, 2021.

Revised Manuscript received on March 22, 2021.

Manuscript published on April 30, 2021.

* Correspondence Author

Nehal Raj*, Student, Department of School of Computer Science and Engineering, Galgotias University, Greater Noida, India

Rajneesh Kumar Singh, Student, Department of School of Computer Science and Engineering, Galgotias University, Greater Noida, India

Satyajee Srivastava, Assistant Professor, Department of School of Computer Science and Engineering, Galgotias University, Greater Noida, India

(c) The Authors. Published by Blue Eyes Intelligence Engineering and Sciences Publication (BEIESP). This is an open access article under the CC BY-NC-ND license (http://creativecommons.org/licenses/by-nc-nd/4.0/) pointed toward expanding the apparent estimation of the buy to the customer. Our objective here is to inspect, utilizing information-driven ML, regardless of whether explicit goal and promptly quantifiable components impact client choices. The particular components which influence a buying choice unavoidably change to a degree starting with one customer then onto the next. This perception has a two-fold impact with regards to the current work. Initially, it proposes that a portion of the prescient force is probably going to be found in segment data on the customer for example the buyer's age, sex, pay, and training. Furthermore, it rouses the utilization of ML with the goal that the impacts of every one of these purchaser explicit factors can be gained from the information. Different factors of interest revolve around the actual item and the way where its buy is introduced. The cost of the item offered, its classification (gadgets, diversion, family merchandise, perishability, and so on) limits, endowments, and other comparative highlights fall inside this gathering of possibly significant factors. Henceforth a blend of outer elements joined with the subtleties prepared at the time the cost of an item is learned structure a bunch of autonomous factors that contextualize buying conduct.

\section{LITERATURE SURVEY}

There are assortments of Algorithmic procedures accessible to perform client purchasing decision dependent on their purchasing conduct and some different qualities moreover.

Van den Poel (2005) [1] uses a random forest Algorithm on a true informational collection to comprehend and foresee three significant proportions of client results: next, purchase incomplete deserting (dropping an item), and clients productivity advancement.An intriguing revelation rising up out of their work was that diverse info factors were found to have the best effect with regards to the three previously mentioned forecasts of interestJuni Nurma Sari et.al in paper [2] creators have characterized the information for client division in two classifications for example Interior information and External information. They had classified the client profile and exchange history information as inside information and information like cookies, worker log, and overview information was arranged in outside information. They have additionally ordered techniques like Magento, Business Rule, Quantile enrollment, Customer Profiling, Supervised clustering, and so on as Simple method, Target procedure, RFM strategy, an Unsupervised procedure. Utilizing Decision trees and regression models Sifa et al. (2015) [3] distinguished various controllable variables of significance -, for example,

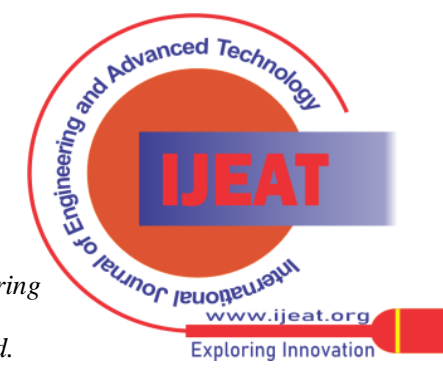




\section{Analysis on Prediction of Customer Purchasing Decisions using Machine Learning}

the quantity of 'associations', 'leisure', and 'area', to give some examples - which gives us better-grained knowledge into what influences a customer's choice to buy. The finding that, for instance, upgrading over boundaries, for example, leisure (for example by making more levels in a game) has the capability of expanding in-game deals (Sifa et al. 2015) can be sensibly expected to have generalizable materialness.

Kareena et.al in their paper [4] proposed a hybrid classifier procedure utilizing a Decision tree and KNN for client conduct examination which beat the exhibition of the recently acknowledged Naïve Bayes model by numerous specialists. The hybrid classifier has a precision of $90.75 \%$ that had a critical distinction from the exactness of the Naïve Bayes classifier that was just $74.11 \%$.A.Salini et.al in paper [5] analyzed the presentation of 3 individual order calculations, for example, random Forest, Support Vector Machine, and Logistic Regression with that of Majority Voting calculation and demonstrated fruitful in accomplishing better Precision, Recall, F-Measure and Accuracy also.

\section{METHODOLOGY}

In this part, we summarize the key specific nuances of the current work. The primary pieces of our informational collection are depicted first, followed by a depiction of the portrayal approaches embraced and the reasons behind our decision. Our information corpus contains 10,000 passages, every one of which compares to a particular buying choice by a shopper for example it is related with a solitary individual and a solitary item under the thought. A definitive result of interest is the choice made by the shopper on whether to buy. Every situation is portrayed by 72 highlights chose as possibly having prescient force in the depicted setting. From now on we will allude to these as B1, ... , B72, and to the objective, class to be anticipated (that is, the buying choice) as Ck. The information has been decontextualized to the importance of every factor has been darkened by hashing. A few factors are consistent and others discrete, some numeric and others text-based. A little illustrative example appears in Table 1 Classification methodologiesFor our tests, we embraced the utilization of two extraordinary, notable characterization draws near. These were essentially chosen based on their far and wide use, surely knew conduct and promising execution in an assortment of other arrangement assignments. In addition, both are promptly relevant on data with heterogeneous highlights, some of which might be unmitigated and some continued, and which may have estimations of limitlessly various reaches (Tun, Arandjelovic,' and Caie 2018). Our objective was additionally to look at classifiers that depend on various suspicions on the connection between various highlights, just as classifiers that contrast as far as the practical types of characterization limits they can learn. The two thought about classifiers are nave Bayes (Jordan 2002; Nigri and Arandjelovic 2017b; Beykikhoshk ' et al. 2015; ) and random forest-based ' classifiers (Breiman 2001; Nigri and Arandjelovic 2017a; ' Barracliffe, Arandjelovic, and Humphris 2017). For culmination, we sum up the critical parts of each next.

\section{Naive Bayes classification}

A Naive Bayes classifier is a probabilistic ML model that is utilized for grouping assignments. The center of the classifier relies upon the Bayes speculation. Naive Bayes classification applies the Bayes hypothesis by making the 'nave' presumption of highlight autonomy. Officially, given a bunch of $n$ highlights $x 1, \ldots, x n$, the related example is considered as having a place with the class $\mathrm{Y}$ which fulfills the

$$
y=\operatorname{argmax}_{y} P(y) \prod_{i=1}^{n} P\left(x_{i} \mid y\right)
$$

accompanying condition:

\section{Random forest}

Random forest, similar to its name infers, comprises an enormous number of individual choice trees that work as an outfit. Every individual tree in the random forest lets out a class forecast and the class with the most votes turns into our model's expectation.Random forest classifiers fall under the expansive umbrella of troupe-based learning strategies (Breiman 2001). They are easy to execute, quick in activity and have demonstrated to be incredibly fruitful in an assortment of areas (Bosch, Zisserman, and Munoz 2007; Cutler et al. 2007; Ghosh and Manjunath 2013). The key guideline basic the random forest approach involves the development of many "straightforward" choice trees in the preparation stage and the greater part vote (mode) across them in the arrangement stage. Among different advantages, this voting methodology amends for the bothersome property of choice trees to overfit preparing data (Zadrozny and Elkan 2001). In the preparation stage, the random forest classifier applies the overall procedure known as sacking (Breiman 1996) to singular trees in the group. Bagging consistently chooses a random instance with substitution from the preparation set and fits trees to these examples. Each tree is developed without pruning. The quantity of trees in the group is a free boundary that is readily learned automatically purported and it is also called out-of-sack mistake (Breiman 2001); this methodology is received in the current work also.

Table1: A little illustrative example of passages in our Data collection contains 10,000 purchaser choices to buy or not to buy a particular item.

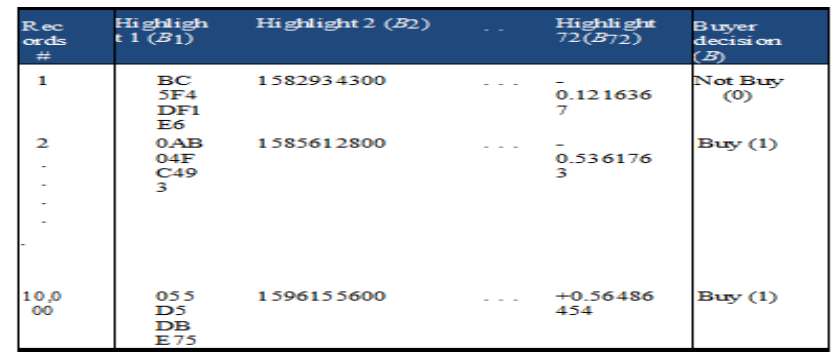

\section{RESULT AND DISCUSSION}

Examinations were performed using the standard 5-cover cross-endorsement show with a ultimate objective to restrict the capacity of overfitting. For the discretionary forest area based classifier we used the forest size of 100 trees, each ready for the most limit significance of 10 .

Published By:

Blue Eyes Intelligence Engineering

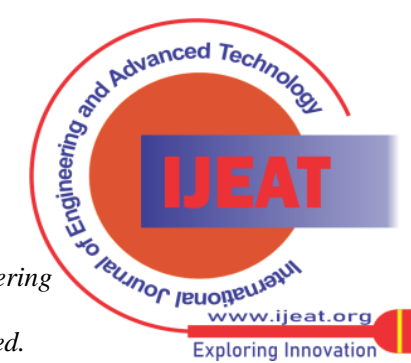


We began our assessment by analyzing and contrasting 'coarse' execution insights of the two classifiers: the normal grouping exactness, the zone under bend (AUC) of the accuracy review trademark, and the F1-score. The primary outcomes are appeared in Table 2. It tends to be promptly seen that the arbitrary timberland based classifier outflanked the basic innocent Bayes approach significantly, the improvement being clear taking all things together execution measures (roughly 10\% improvement for each situation). More nuanced understanding can be acquired by looking at the disarray frameworks relating to the two techniques these are appeared in Figure 3. What is fascinating to see from this figure is that the strategies performed almost indistinguishably when the buying choice was negative (for example no buy was made). The exhibition improvement saw by the insights in Table 2 can be believed to rise out of forecasts identifying with cases when the client decided to seek after a buy. Taking into account that our information is adjusted as far as the portrayal of the two classes (see past segment and Figure 2 specifically), this marvel can't be clarified because of an antiquity in the information. Or maybe the clarification must be that the collaboration of various highlights depicting the buying setting interface in a more nuanced way when the client proceeds with the buywhich can be caught by a more mind boggling classifier, for example, one dependent on an irregular woods however not by a simple naive Bayes approach Specifically, considering the principal presumption supporting the last mentioned (review that the interpretability of characterization was one of our purposes behind choosing these particular classifiers, as depicted in the past area) we are led to conclude that there is a greater degree of interaction and a decrease of independence between features when the customer makes a positive purchasing decision. This explanation also resonates with our intuition: a decision to purchase implies a financial commitment and a loss of money, motivating a more in-depth thought process. Indeed, this explanation is further corroborated by the analysis of the importance of different features summarizedin figure 2. Significance was evaluated utilizing the standard methodology presented by Breiman (2001) which depends on the age of arbitrary changes of highlights and an examination of the outcomes utilizing such highlights with a prepared backwoods. The significant perception to take from this figure concerns the mistake bars (for example the standard deviations) which are extremely expansive. This recommends, proving our past perceptions, that there is a serious level of excess between various highlights. At last, we delineated this by per-shaping an element choice interaction, and looking at order execution utilizing a decreased arrangement of highlights with the outcomes point by point prior, utilizing the whole information space. Specifically, we embraced an iterative methodology whereby (I) the main component was found utilizing Breiman's strategy (ii) the element was chosen and subsequently eliminated from the accessible set, and (iii) the significance of the leftover highlights reconsidered. This is as a result an eager way to deal with include choice. Our outcomes are summed up in Table 3 . As the insights in the table make clear, the information include set was diminished by $70 \%$ (from 72 to 22 ) essentially with no negative impact on characterization execution regarding normal arrangement precision, AUC, and F1-score.Table 2: A rundown of the key 'coarse' execution measurements of the two classifiers utilized in our investigations. It very well may be promptly seen that the random forest based classifier beat the basic naiıe Bayes approach considerably, the improvement being obvious taking all things together execution measures (roughly 10\% improvement for each situation).

\begin{tabular}{|c|l|l|}
\hline Measure & Naive Bayes & Random forest \\
\hline Accuracy & 0.66 & 0.72 \\
\hline AUC & 0.71 & 0.79 \\
\hline F1-score & 0.66 & 0.72 \\
\hline
\end{tabular}

\section{CONCLUSION}

In this paper we examined the test of anticipating purchaser buying choices utilizing promptly quantifiable highlights of the buying setting. Differentiating past work, in this we didn't limit our thoughtfulness regarding a particular item classification, retailer type, or client segment, yet rather utilized an enormous and assorted informational index gathered in 'this present reality' from genuine client item connection occasions. In addition, our methodology is altogether information driven and not at all like most existing examination in the field, doesn't utilize any abstract decisions or deduced presumptions. Adding to the significance of our work is the way that the informational collection utilized in the analyses we depict is, to the most amazing aspect our insight, the biggest one utilized in the distributed, peer looked into, academic writing. Our outcomes give various novel experiences into shopper conduct, among others proof of various perspectives occurring in the committal purchasing activity from those hidden the traditionalist choice not to proceed with the buy. The introduced discoveries and the going with conversation feature roads for future exploration,givesignificant information both to shoppers, and retailers and specialistco-ops.

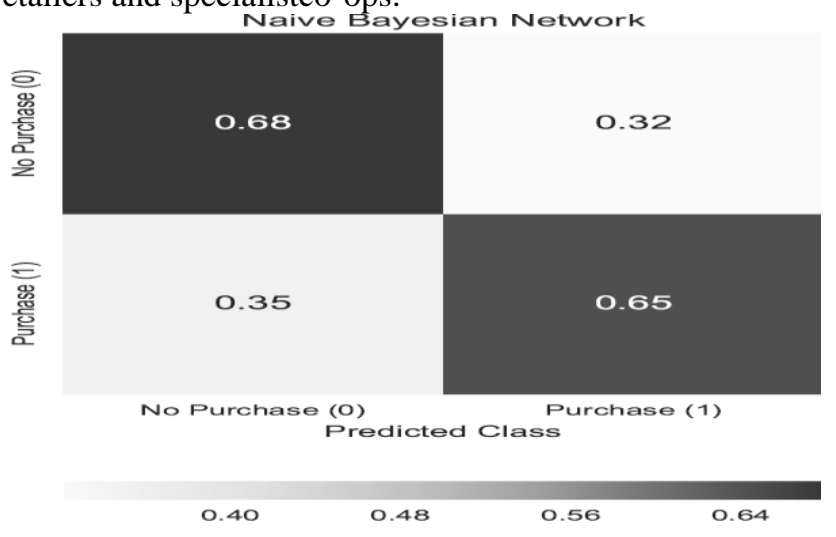

\section{A). NAIVEBAYES}

Figure 1: Confusion grids relating to the nave Bayes (left) and random forest(right) based classifiers. See that the techniques performed almost indistinguishably when the buying choice was negative (for example no buy was made). The exhibition improvement saw by the insights in Table 2 can be believed to rise up out of forecasts identifying with examples when the client decided to seek after a buy.

Published By:

Blue Eyes Intelligence Engineering \& Sciences Publication 


\section{Analysis on Prediction of Customer Purchasing Decisions using Machine Learning}

This proposes that there is a more prominent level of association and a lessening of freedom between highlights when the client settles on a positive buying choice

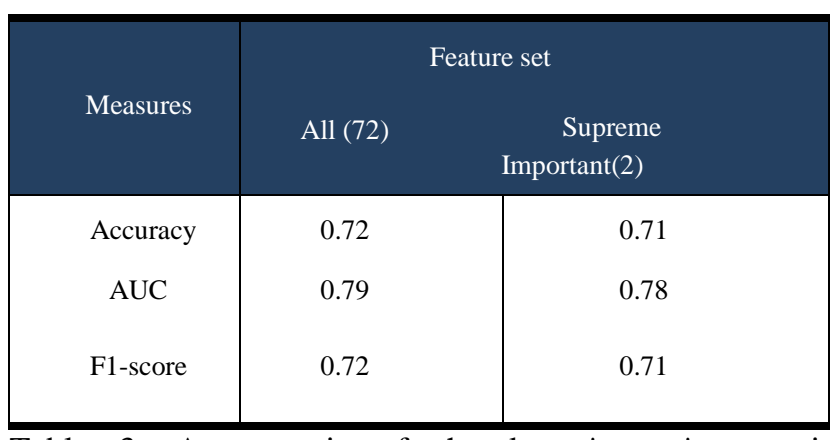

Table 3: A synopsis of the key 'coarse' execution measurements of the irregular woods based classifier looking at its presentation when all accessible info highlights are utilized (72 altogether) versus utilizing the 22 most significant highlights just, chose in an insatiable style with significance reexamination each time a component is chosen.

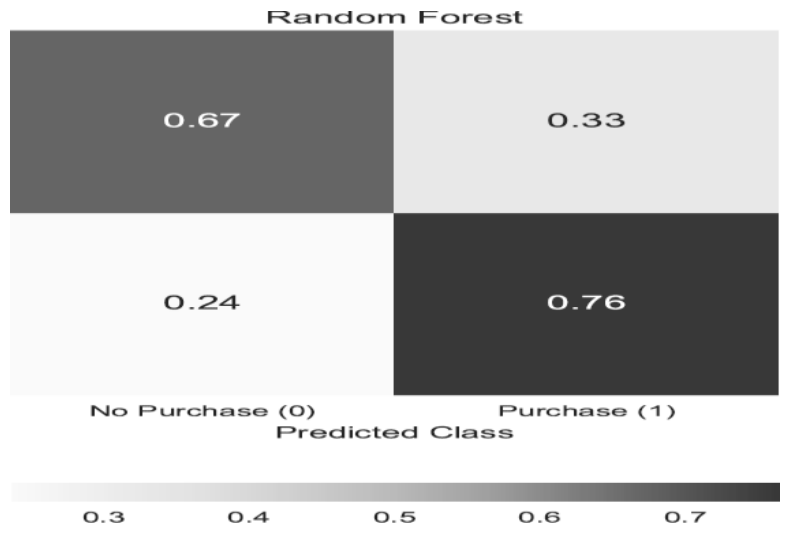

\section{B) RANDOM FOREST}

\section{REFERENCES}

1. Juni Nurma Sari, Lukito Nugroho, Ridi Ferdiana, Paulus Insap Santosa: Review on Customer Segmentation Technique on Ecommerce, Journal of Computational and Theoretical Nanoscience, 22(10):3018-3022, Oct-2016

2. Jing Wu, Zheng Lin: Research on customer Puchasing Prediction model by clustering, ICEC'05, Proceedings of the 7th International conference on Electronic commerce, Pag316- 318, Aug- 2005, DOI:10.1145/1089551.1089610

3. Kareena, Raj Kumar: A Consumer Behavior PredictionMethod for E-Commerce Application, International Journal of Recent Technology and Engineering (IJRTE), ISSN: 2277-3878, Volume- 8, Issue - 2S6, Jul-2019

4. A.Salini, U.Jeyapriya: A Majority Vote Based Ensemble Classifier for Predicting Students Academic Performance, International Journal of Pure and Applied Mathematics, Volume 118 No. 24, ISSN: 1314-3395, Mar-2018.

5. B.B.Goyal, Meghna Aggarwal:Organized retailing in India - An empirical study ofappropriate formats and expected trends,Global Journal of Business Research,Volume 3,Number 2,2009.

6. Market Segmentation, Targeting and Positioning: Travel Marketing, Tourism Economics and the Airline Product: An Introduction to Theory and Practice, Edition:1, Chapter: 4, Springer, Dec- 2017, DOI: 10.1007/978-3-319-49849-2_4

7. Machine Learning Based Prediction of Consumer Purchasing Decisions The Evidenceand Its Significance Saavi Stubseid and Ognjen Arandjelovic School of Computer Science University of St Andrew St Andrews, Fife, KY16 9SX United Kingdom.

8. Yuanlin Chen, Yueting Chai, Yi Liu, and YangXu, "Analysis of Review Helpfulness Based onConsumer Perspective", Tsinghua Science and Technology, 2015, Volume: 20, Issue: 3,Page s: 293 -.

Published By:

Blue Eyes Intelligence Engineering

Rajneesh Kumar Singh, is a data science enthusiast with a strong inclination towards problem-solving and propelling data-driven decisions. He is currently pursuing his Bachelor of Technology in Computer Science Engineering from Galgotias University. He has worked on multiple projects in the field of machine learning.He is a keen learner of new Technologies. .He has also attended multiple workshops on Data Science His field of research is machine learning, E-mail: 2018rajneeshsingh@gmail.com

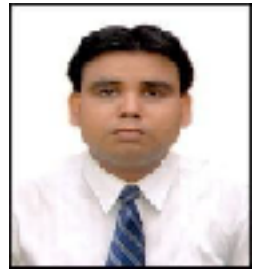

Dr. Satyajee Srivastava, has over 17 years of teaching and industry experience. He received his Ph.D. in Computer Science and is an Artificial Intelligence developer, researcher, practitioner, and educator. He has Publication of 8 Books and 35 Articles in Journals of repute He is presently serving as Associate Professor at Galgotias University Greater Noida He is an active machine learning researcher and regularly teachcourses and maintains resources for the data scientist. He is a member of the technical program committees for several technical conferences and editorial member of reputed journals. He has chaired, participated in and presented at conferences and seminars in India and across the world. Apart from academic pursuits, he has shouldered many administrative responsibilities in various capacities. His research has pioneered developments in ensemble learning, outlier detection and profile discovery Data Science Data Mining ,Big DATA and Green Computing. Email: drsatyajee@gmail.com

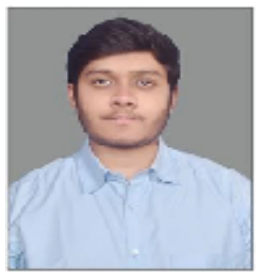

Nehal Raj,is an aspiring learner. He has Good knowledge of Data Science andMachine Learning with a strong inclination towards problem-solving and propelling data-driven decisions. $\mathrm{He}$ is currently pursuing his Bachelor of Technology in Computer Science Engineering from Galgotias University. He has worked on multiple projects related to problem solving and Data science in his college Academics. He has also attended multiple workshops on Machine Learning during his college. His field of research is machine learning and data science.

Email:nehalraj27@gmail.com 